\title{
Correction to: Effect of Gestational Exposure of Cypermethrin on Postnatal Development of Brain Cytochrome P450 2D1 and 3A1 and Neurotransmitter Receptors
}

\author{
Anshuman Singh ${ }^{1,2} \cdot$ Anubha Mudawal $^{1} \cdot$ Rajendra K. Shukla $^{1}$. Sanjay Yadav ${ }^{1}$. Vinay K Khanna ${ }^{1}$. \\ Rao Sethumadhavan ${ }^{2} \cdot$ Devendra Parmar ${ }^{1}$
}

Published online: 2 September 2019

(C) Springer Science+Business Media, LLC, part of Springer Nature 2019

Correction to: Mol Neurobiol (2015) 52:741-756

https://doi.org/10.1007/s12035-014-8903-6

The original version of this article unfortunately contained errors in Fig. 4a. Representative image of b-actin of brain region were copied incorrectly during the preparation of the figures.

The online version of the original article can be found at https://doi.org/ 10.1007/s12035-014-8903-6

Devendra Parmar

parmar_devendra@hotmail.com

Anshuman Singh

anshuman321_80@hotmail.com

Anubha Mudawal

anubha213@gmail.com

Rajendra K. Shukla

razshukla@gmail.com

Sanjay Yadav

sanjayitrc@gmail.com
Vinay K Khanna

vkkhanna1@gmail.com

Rao Sethumadhavan

rsethumadhavan@vit.ac.in

Developmental Toxicology Division, CSIR - Indian Institute of Toxicology Research, Post Box No. 80, M.G. Marg, Lucknow, Uttar Pradesh 226001, India

2 School of Bio Sciences and Technology, Vellore Institute of Technology, Vellore, Tamil Nadu 632014, India 
The corrected Figure is given below.

a

GABA $_{\text {Aa1 }}$

CHRM2

DA-D2

5-HT2A

$\beta$-actin

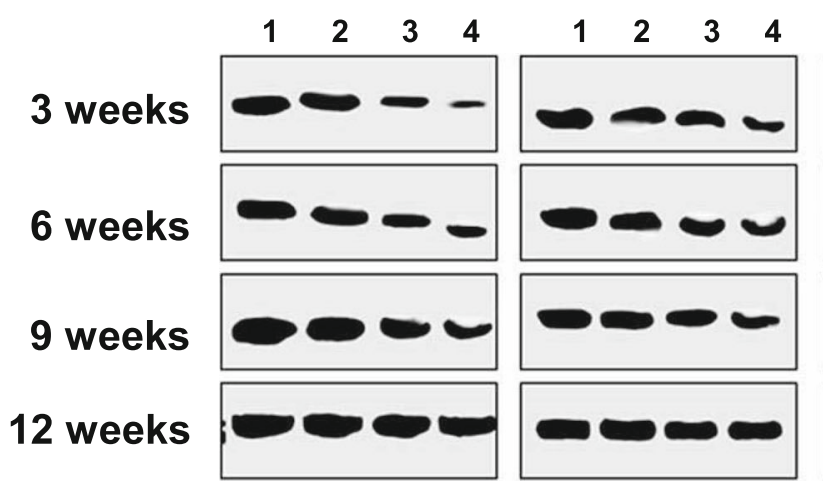

Fig. 4 a Representative western blots for synaptosomal proteins isolated from brain regions of prenatally exposed offsprings with anti-GABA (cerebellum) or CHRM2 (hippocampus) or DA-D2 (corpus striatum) or 5-HT2A (frontal cortex). Lane 1 contains synaptosomal proteins $(50 \mu \mathrm{g})$ from cerebellum or hippocampus or corpus striatum or frontal cortex of
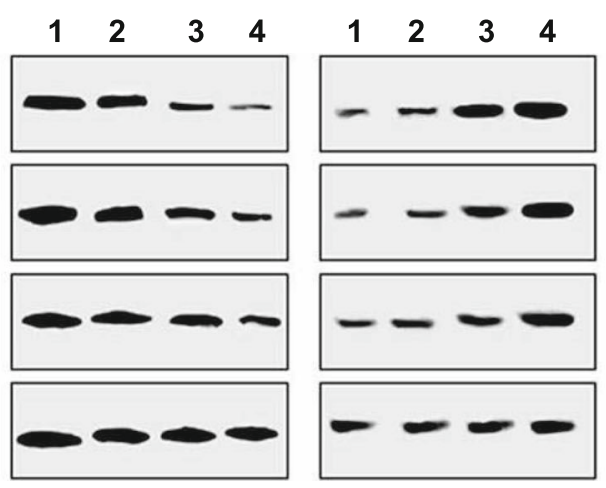

offsprings raised on control rat mothers. Lanes 2-4 contain synaptosomal proteins $(50 \mu \mathrm{g})$ from cerebellum or hippocampus or corpus striatum or frontal cortex of offsprings exposed prenatally to $1.25,2.5$ and $5 \mathrm{mg} / \mathrm{kg}$ of cypermethrin

The replacement of figure will not affect the outcome of study.

Publisher's Note Springer Nature remains neutral with regard to jurisdictional claims in published maps and institutional affiliations. 